\title{
Could Reduced Cerebrospinal Fluid (CSF) Galanin Contribute to Restricted Eating in Anorexia Nervosa?
}

\author{
Guido K. Frank, M.D., Walter H. Kaye, M.D., Abhiram Sahu, Ph.D., John Fernstrom, Ph.D., \\ and Claire McConaha, B.S.N.
}

\begin{abstract}
Galanin (GAL) and gamma amino butyric acid (GABA) are orexigenic neuropeptides that could contribute to the pathogenesis of anorexia nervosa (AN). To avoid the confounding effects of the ill state, we studied women who were recovered ( $>1$ year, normal weight, and regular menstrual cycles, no bingeing or purging) from AN (REC $A N)$ and matched healthy control women (NC). CSF GAL was reduced in REC AN $(64.4 \pm 8.6 \mathrm{pg} / \mathrm{ml})$ compared to NC $(72.0 \pm 11.6 \mathrm{pg} / \mathrm{ml} ; p<.05)$, GABA was similar
\end{abstract}

between groups. In the brain, GAL stimulates appetite and fat consumption. These data raise the question of whether alterations in brain GAL activity plays a role in clinical symptoms in AN, such as food restriction and fat avoidance. [Neuropsychopharmacology 24:706-709, 2001] (C) 2001 American College of Neuropsychopharmacology. Published by Elsevier Science Inc.
KEY WORDS: Anorexia nervosa; Bulimia nervosa; Recovery; Galanin; GABA

Anorexia nervosa (AN) is a disorder of unknown etiology (American Psychiatric Association 1994). The most distinguishing characteristic of $\mathrm{AN}$ is severe emaciation. Two subtypes have been identified. Restrictingtype AN patients lose weight by pure restricted eating. Bulimic anorexics also restrict food, but engage episodically in inappropriate compensatory behaviors, most often repeated bingeing and purging behavior. It has been hypothesized that a disturbance of brain neurotransmitter function might contribute to disturbances of food ingestion in AN. Two neuropeptides of potential

From the Department of Psychiatry, Western Psychiatric Institute and Clinic (GKF, WHK, JF, CM) and the Department of Cell Biology and Physiology, Biomedical Science Tower (AS), University of Pittsburgh, School of Medicine, Pittsburgh, PA.

Address correspondence to: Walter H. Kaye, M.D., University of Pittsburgh, Western Psychiatric Institute and Clinic, 3811 O'Hara Street, Room E-724, Pittsburgh, PA 15213.

Received 27 June 2000; revised 5 November 2000; accepted 14 November 2000. interest are galanin (GAL) and gamma amino butyric acid (GABA) since both peptides contribute to the modulation of feeding (Hoebel 1997; Kelley 1999).

GAL and GABA are expressed in several hypothalamic sites and they are co-expressed in the hypothalamus (HTL) arcuate nucleus (Kalra et al. 1999). The main site of action of brain GAL is the paraventricular nucleus of the HTL as well as other HTL nuclei (Crawley 1999). GAL may stimulate appetite (Lee et al. 1994) and in particular fat intake (Leibowitz 1995). GABA has been suggested to be an important part of a system in the brain that constitutes "taste pleasure" (Berridge and Pecina 1995), and may transmit taste information (Yamamoto et al. 1998).

Disturbances of neuropeptides could be a consequence of disturbed eating behavior or malnutrition, or pre-morbid traits that contribute to a vulnerability to develop an ED. Determining whether abnormalities are a consequence or a potential antecedent of pathological feeding behavior is a major question in the study of eating disorders. It is impractical to study people with ED prospectively due to the young age of onset and diffi- 
culty in premorbid identification of people who will develop an ED. Therefore, we decided to study women who had recovered for more than a year from AN. Any persistent psychobiological abnormalities might be traitrelated and potentially contribute to the pathogenesis of this disorder. In this study we measured CSF values of GAL and GABA in subjects who were long-term recovered from AN (REC AN). We hypothesized that REC AN might have decreased GAL or GABA activity which might contribute to reduced motivation to eat.

\section{METHODS}

Subjects were recruited, screened and diagnosed as previously described (Kaye et al. 1998, 1999). Diagnoses were adapted to DSM-IV criteria. REC AN subjects were 18 women who had previously met DSM-IV criteria for $\mathrm{AN}, 13$ women who had recovered from binge eating/purging, and five women who had recovered from restricting type AN. Recovered ED subjects were compared to 16 female normal controls (NC). In the year before the study, all participating recovered subjects had to maintain a body weight of $>90 \%$ average body weight (\% ABW) (Metropolitan Life Insurance 1959); have regular menstrual cycles; have not binged, purged, or engaged in restrictive eating patterns; and have not met criteria for substance-related disorders. Subjects were free of medication for 6 weeks prior to the study and gave informed consent. The NC had no history of an eating disorder or any psychiatric, medical, or neurologic illness. They had normal menstrual cycles, and had been within a normal weight range since menarche. They also had no first degree relatives with an eating disorder.

A lumbar puncture (LP) for obtaining CSF samples was performed during the early follicular phase of the most recent menstrual cycle by methods previously described (Kaye et al. 1998). For the CSF GAL assay a commercial kit for radio immunoassay (RIA) from Peninsula Laboratories, Inc., San Carlos, CA, was used. CSF GABA concentrations were estimated using a BAS MF9054 Amino Acid Analysis Kit (Bioanalytical Systems, Inc., West Lafayette, IN). Plasma $\beta$-hydroxy butyrate (BHBA), a ketone body, was assessed as an index of starvation. BHBA was measured by the enzymatic method of Williamson et al. (1962).

Statistical analyses were done using the SPSS software package (SPSS, Chicago, IL) (Barcikowski 1984). Correlations were investigated using Spearman Correlation coefficients. Two tailed independent samples $\mathrm{t}$-tests investigated group differences. If outcome variables showed significant correlations with demographic variables, analysis of covariance (ANCOVA) assessed between group differences using such variables as covariates.
Table 1. Demographic Variables and Neuropeptide Values

\begin{tabular}{lccrl}
\hline & $\begin{array}{c}\text { NC } \\
(\boldsymbol{n}=\mathbf{1 6})\end{array}$ & $\begin{array}{c}\text { REC AN } \\
(\boldsymbol{n}=\mathbf{1 8})\end{array}$ & $\mathbf{t}$ & \multicolumn{1}{c}{$\boldsymbol{p}$} \\
\hline Age (years) & $22.9 \pm 4.4$ & $24.5 \pm 3.2$ & -1.20 & 0.24 \\
Weight (kg) & $59.6 \pm 5.4$ & $53.6 \pm 5.6$ & 3.06 & $0.01^{*}$ \\
\%ABW & $104 \pm 8$ & $97 \pm 9$ & 2.31 & $0.03^{*}$ \\
HIGH \%ABW & $110 \pm 9$ & $109 \pm 19$ & 0.25 & 0.80 \\
LOW \%ABW & $97 \pm 9$ & $69 \pm 8$ & 9.02 & $0.00^{*}$ \\
BHBA & $113.7 \pm 82.2$ & $188.5 \pm 138.5$ & -1.68 & 0.11 \\
Galanin $(\mathrm{pg} / \mathrm{ml})$ & $72.0 \pm 11.6$ & $64.4 \pm 8.6$ & 2.17 & $0.04^{*}$ \\
GABA $(\mathrm{pmol} / \mathrm{ml})$ & $39.4 \pm 18.5$ & $41.9 \pm 15.3$ & -0.35 & 0.73
\end{tabular}

Note: All values are expressed as mean \pm standard deviation.

$\% \mathrm{ABW}, \%$ average body weight; BHBA, $\beta$-hydroxybutyric acid; GABA, gamma amino butyric acid; $\mathrm{kg}$, kilograms; $\mathrm{t}$, Two tailed independent samples t-tests.

\section{RESULTS}

Age, high \% ABW, and BHBA were similar between groups. Current and past low \% ABW were significantly lower in REC AN (Table 1). Compared to CW, AN women had similar CSF GABA (Table 1). However, CSF GAL concentrations were significantly lower in REC AN (Figure 1). CSF GAL concentrations were similar for restricting type REC AN $(n=5,62.1 \pm 3.6 \mathrm{pg} /$ $\mathrm{ml})$ and binge eating/purging type $\operatorname{REC} \mathrm{AN}(n=13$, $65.3 \pm 9.8 \mathrm{pg} / \mathrm{ml})(\mathrm{t}=0.7, p=.5)$; an additional nonparametric Mann-Whitney $U$ test showed a similar result (exact significance $=0.04$ ). The range of CSF GAL was almost similar for both groups (NC: $39 \mathrm{pg} / \mathrm{ml}$ vs. REC AN:41 pg/ml), whereas the minimum $(58 \mathrm{pg} / \mathrm{ml})$

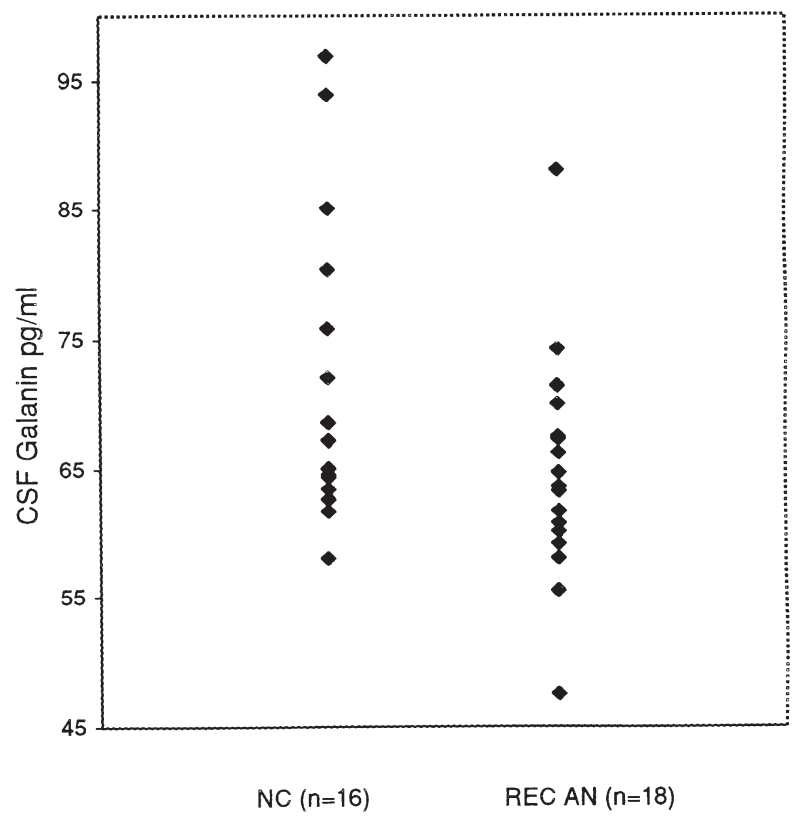

Figure 1. Scatterplot of galanin distribution within and between groups. 
and maximum $(97 \mathrm{pg} / \mathrm{ml})$ for the $\mathrm{NC}$ group were higher than for the REC AN women (minimum $47 \mathrm{pg}$ / $\mathrm{ml}$, maximum $88 \mathrm{pg} / \mathrm{ml}$. In addition, the variance was higher for NC women (135 pg/ml) compared to REC AN $(73 \mathrm{pg} / \mathrm{ml})$.

There was no significant correlation between GAL and any of the demographic variables, including plasma BHBA, in either one of the two groups. CSF GABA was significantly correlated with current $\%$ ABW (rho $=-0.68, p<.01$ ) in NC, but not REC AN women (rho $=-0.31, p=.39$ ). An ANCOVA assessing GABA using current $\% \mathrm{ABW}$ as covariate, still showed similar GABA values between groups $(\mathrm{F}=0.64, p=.43)$.

\section{DISCUSSION}

This study found reduced CSF concentrations of GAL but normal CSF GABA levels in women who were long term recovered from AN. In the past, ill anorexic subjects had normal concentrations of CSF GABA (Gerner and Hare 1981). A previous study from our group (Berrettini et al. 1988) reported that CSF GAL levels were normal in AN women who were studied after being weight restored for more than six months. However, the majority of the subjects in the earlier study had irregular or absent menstrual cycles, and a substantial proportion were below $90 \%$ ideal body weight. Thus, most study subjects were still in the process of recovery. The reasons for the differences in CSF GAL concentrations in these two studies remains uncertain. Whether this is a phenomenon that only emerges after long-term recovery is possible, and deserves further study.

The differences between subject groups was modest (Figure 1) although significantly different. The meaning of altered concentrations of CSF neuropeptides levels in humans is not certain. It is possible that such modest findings in the CSF could reflect a disturbance at the receptor or intracellular translational pathways, which may have a substantial effect on feeding behavior but only have modest effects on neuropeptide concentrations. Whether differences in weight or nutritional status account for the discrepancy between our study and that of Berrettini et al. (1988) is not known. Studies in animals suggests that restricted eating behavior does not alter GAL concentrations in the HTL (Crawley 1999). However, reductions of leptin, which is well know to be decreased in the ill state of AN, could theoretically down regulate central GAL mRNA (Sahu et al. 1998a, 1998b) after recovery and thus account for state differences. Alternatively, GAL is co-expressed with 5-HT in dorsal raphe neurons (Hokfelt et al. 1999), and may inhibit 5-HT secretion (Fuxe et al. 1998). Ill AN have low concentrations of CSF 5-HIAA, the major brain serotonin metabolite, whereas elevated concentration are found after long term recovery (Kaye et al. 1991). While the subjects in this study had elevated CSF 5-HIAA (data not shown), no significant relationship with CSF GAL was found. In the brain, GAL stimulates appetite and in particular fat consumption (Lee et al. 1994; Leibowitz 1995). These data raise the question of whether alterations in brain GAL activity play a role in common clinical symptoms in AN, such as food restriction and fat avoidance (Sunday and Halmi 1990).

An animal study (Podell and Hadjiconstantinou 1997) also found a negative correlation of GABA in CSF with body weight. The meaning of a correlation between GABA and \% ABW is not clear.

In terms of limitations, the mean differences for CSF GAL between groups was modest but significant. The relatively small sample size limited our power of observation, and we did not correct for multiple comparisons, for this is an exploratory study and a small sample. Only five of the REC AN women were pure food restrictors, the remaining REC AN subjects were binge eating-purging type AN. It remains uncertain whether CSF values of these compounds actually reflect secretory activity in the brain, or other factors, such as compound metabolism. Finally, it is possible that persistent disturbances of CSF GAL could be a "scar" that is secondary to years of malnutrition or other state related factors. The design of this study, however, does not permit answering this question and this will require a longitudinal study design.

In summary, reduced CSF GAL in long term recovered AN raises the possibility that this is a trait related disturbance and should be further studied during various stages of recovery. Such a disturbance could contribute to food avoidance and perhaps fat aversion in $\mathrm{AN}$.

\section{ACKNOWLEDGMENTS}

Supported in part by grants from NIMH \# 2 R01 MH 42984-04 "The Neurobiology of Feeding Behavior in Bulimia;" Children's Hospital Clinical Research Center, Pittsburgh PA (\# 5M01RR00084); NIMH \# 2 R01 MH 46001-07A1 "Serotonin A Trait Disturbance in Anorexia Nervosa," and NIH HD24730 (Dr. J. Fernstrom).

\section{REFERENCES}

American Psychiatric Association (1994): Diagnostic and Statistical Manual of Mental Disorders, 4th ed. Washington, DC, American Psychiatric Press

Barcikowski RS (1984): Computer Packages and Research Design, Vol 3. SPSS \& SPSSX. Lanham, MD, University Press of America

Berridge KC, Pecina S (1995): Benzodiazepines, appetite, and taste palatability. Neurosci Biobehav Rev 19(1):121131 
Berrettini WH, Kaye WH, Sunderland T, May C, Gwirtsman HE, Mellow A, Albright A (1988): Galanin immunoreactivity in human CSF: studies in eating disorders and Alzheimer's disease. Neuropsychobiology 19(2):64-68

Crawley JN (1999): The role of galanin in feeding behavior. Neuropeptides 33(5):369-375

Fuxe K, Jansson A, Diaz-Cabiale Z, Andersson A, Tinner B, Finnman UB, Misane I, Razani H, Wang FH, Agnati LF, Ogren SO (1998): Galanin modulates 5-hydroxytryptamine functions. Focus on galanin and galanin fragment/5-hydroxytryptamine1A receptor interactions in the brain. Ann N Y Acad Sci 863:274-290

Gerner RH, Hare TA (1981): CSF GABA in normal subjects and patients with depression, schizophrenia, mania, and anorexia nervosa. Am J Psychiatry 138(8):10981101

Hoebel BG (1997): Neuroscience and appetitive behavior research: 25 years. Appetite 29(2):119-133

Hokfelt T, Broberger C, Diez M, Xu ZQ, Shi T, Kopp J, Zhang X, Holmberg K, Landry M, Koistinaho J (1999): Galanin and NPY, two peptides with multiple putative roles in the nervous system. Hormone Metab Res 31(5):330-334

Kalra SP, Dube MG, Pu S, Xu B, Horvath TL, Kalra PS (1999): Interacting appetite-regulating pathways in the hypothalamic regulation of body weight. Endocr Rev 20(1):68-100

Kaye WH, Gwirtsman HE, George DT, Ebert MH (1991): Altered serotonin activity in anorexia nervosa after long-term weight restoration. Does elevated cerebrospinal fluid 5-hydroxyindoleacetic acid level correlate with rigid and obsessive behavior? Arch Gen Psychiatry 48(6):556-562

Kaye WH, Greeno CG, Moss H, Fernstrom J, Fernstrom M, Lilenfeld LR, Weltzin TE, Mann JJ (1998): Alterations in serotonin activity and psychiatric symptoms after recovery from bulimia nervosa. Arch Gen Psychiatry 55(10):927-935
Kaye WH, Frank GK, McConaha C (1999): Altered dopamine activity after recovery from restricting-type anorexia nervosa. Neuropsychopharmacology 21(4):503-506

Kelley AE (1999): Functional specificity of ventral striatal compartments in appetitive behaviors. Ann N Y Acad Sci 877:71-90

Lee MC, Schiffman SS, Pappas TN (1994): Role of neuropeptides in the regulation of feeding behavior: A review of cholecystokinin, bombesin, neuropeptide $\mathrm{Y}$, and galanin. Neurosci Biobehav Rev 18(3):313-323

Leibowitz SF (1995): Brain peptides and obesity: Pharmacologic treatment. Obes Res 3(Suppl 4):573S-589S

Metropolitan Life Insurance Company (1959): New weight standards for men and women, 40. Stat Bull Metrop Insur Co

Podell M, Hadjiconstantinou M (1997): Cerebrospinal fluid gamma-aminobutyric acid and glutamate values in dogs with epilepsy. Am J Vet Res 58(5):451-456

Sahu A (1998a): Evidence suggesting that galanin (GAL), melanin-concentrating hormone $(\mathrm{MCH})$, neurotensin (NT), proopiomelanocortin (POMC) and neuropeptide Y (NPY) are targets of leptin signaling in the hypothalamus. Endocrinology 139(2):795-798

Sahu A (1998b): Leptin decreases food intake induced by melanin-concentrating hormone $(\mathrm{MCH})$, galanin (GAL) and neuropeptide $\mathrm{Y}$ (NPY) in the rat. Endocrinology 139(11):4739-4742

Sunday SR, Halmi KA (1990): Taste perceptions and hedonics in eating disorders. Physiol Behav 48(5):587-594

Williamson DH, Mellanby J, Krebs HA (1962): Enzymatic determination of $\mathrm{D}(-) \beta$ hydroxybutyric acid and acetoacetic acid in blood. Biochem J 82:90-96

Yamamoto T, Nagai T, Shimura T, Yasoshima Y (1998): Roles of chemical mediators in the taste system. Jap J Pharmacol 76(4):325-348 